\title{
Infecciones respiratorias agudas bajas en niños: Estudio etiológico prospectivo
}

\author{
JAIME LOZANO C.***, LETICIA YÁNEZ P.**, MICHELANGELO LAPADULA A.**, \\ MÓNICA LAFOURCADE R.***, FELIPE BURGOS F.****, \\ ISOLDA BUDNIK O.***** y LUIS HERRADA H.*****
}

\section{Lower respiratory tract infections in children: A prospective etiological study}

Background: Lower respiratory tract infection (LRTI) is a serious cause of morbidity and admission among children. The aim of the present investigation was to determine the etiology of severe LRTI in hospitalized children by several laboratory methods in a systematic way. Methods: A 2-year prospective study. Results: A potential causative agent was detected in 155(68\%) of the 229 patients. A viral infection was identified in 60\%, $15 \%$ had bacterial infection and $4 \%$ had evidence of concomitant viral-bacterial infection. Virus, with the predominance of respiratory syncytial virus $(R S V)$ was the most common agent in children younger than 24 month. Mycoplasma pneumoniae and Streptococcus pneumoniae were the most important bacteria. Conclusions: The possible causative agent on childhood LRTI could be detected in most cases. The data confirm the importance of virus especially RSV in the LRTI in hospitalized children.

Key words: Acute respiratory tract infections; hospitalized children; etiological agents.

\section{Resumen}

Resumen: Las infecciones respiratorias agudas bajas (IRAb) son causa importante de morbilidad $y$ de hospitalización en los niños. El objetivo de esta investigación fue determinar la etiología de IRAb grave de niños hospitalizados por varios métodos diagnósticos en forma protocolizada. Método: Estudio prospectivo en dos años consecutivos. Resultados: Un posible agente causal fue detectado en 155 (68\%) de 229 pacientes: Infección viral fue identificada en 60\%, bacteriana: 15\% y mixta: 4\%. Virus, principalmente virus respiratorio sincicial (VRS) fue el agente etiológico más frecuente en menores de 24 meses. Mycoplasma pneumoniae y Streptococcus pneumoniae fueron las principales bacterias aisladas. Conclusiones: Un posible agente causal de la IRAb en niños pudo ser identificado en la mayoría de los casos. Los datos confirman la importancia de los virus, especialmente VRS, en la IRAb en niños hospitalizados.

Palabras clave: Infecciones respiratorias agudas; niños hospitalizados, agentes etiológicos.

\section{Introducción}

Las infecciones respiratorias agudas que comprometen las vías respiratorias inferiores en niños (IRAb) son una causa frecuente de morbilidad y de hospitalización $n^{1,3,5-7,13,14,16}$. Establecer la etiología es importante en situaciones como: neumonía grave e insuficiencia respiratoria ${ }^{1}$. También para la formulación de recomendaciones terapéuticas ${ }^{2-4,15}$, aislamiento del paciente, evaluación de la efectividad de las vacunas en uso actualmente y para la elaboración de nuevas va-

\footnotetext{
Clínica Santa María:

* Broncopulmonar.

** Unidad de Paciente Crítico Pediátrico.

*** Microbiólogo.

**** Kinesiólogo.

******Internos de Medicina, Universidad de Los Andes.
} 
cunas $^{1,2,11,12}$. Un gran número de microorganismos pueden causar una IRAb. Determinar la etiología puede resultar difícil por varias razones ${ }^{1,4}$. El estudio para bacterias en los niños tiene limitaciones especialmente debido a dificultades en la obtención de una muestra adecuada $^{1,4,5}$. Para virus, los exámenes disponibles permiten identificar sólo a algunos y además la sensibilidad no es buena.

Un número limitado de estudios prospectivos que emplean diversos métodos diagnósticos, logran la identificación etiológica entre el 40 y $85 \%$ de los $\operatorname{casos}^{1-3}$. Mientras más exámenes diagnósticos sean realizados, el número de potenciales agentes causales aumenta ${ }^{1,4,5}$. Nuevas técnicas diagnósticas han permitido identificar virus como Metapneumovirus humano (hMPV) ${ }^{4,6-9}$ y redefinir el rol de otros. Rhinovirus $(\mathrm{RV})^{4}$ antes considerado responsable sólo de IRA altas, hoy en día se asocia con neumonía adquirida en la comunidad, bronquiolitis y exacerbación de asma bronquial.

Realizamos un estudio prospectivo durante 2 años consecutivos en niños hospitalizados por una IRAb en la Unidad de Paciente Crítico Pediátrico de la Clínica Santa María (UPCPCSM) para establecer, con los métodos disponibles un potencial agente causal.

\section{Métodos}

Población estudiada: 229 pacientes hospitalizados consecutivamente en la UPCPCSM por una IRAb, se evaluaron propectivamente en un período de 2 años, entre el 31 de mayo de 2005 y el 31 de mayo de 2007. Se incluyeron niños inmunocompetentes, entre 2 semanas y 15 años. $\mathrm{Al}$ ingreso, a cada paciente se le practicó una evaluación clínica, Rx de tórax y dentro de las primeras $48 \mathrm{~h}$ el siguiente protocolo de estudio etiológico: dos muestras de sangre para hemocultivos, hemograma, VHS, PCR y detección de IgM en suero para Mycoplasma pneumoniae mediante enzimo-inmunoensayo con Inmunocard $\mathrm{R}$ (Meridian Bioscience USA Cincinnati Ohio 45244), cultivo de líquido pleural (si procedía) y dos muestras de secreciones obtenidas por aspirado nasofaríngeo: una para la detección antigénica de virus respiratorio sincicial (VRS), Adenovirus (ADV), Influenza A y B y Parainfluenza 1, 2 y 3 por inmunofluorescencia directa (IFD) empleando anticuerpos monoclonales (Kit Respiratory Virus Panel PathoDx, Lenexa, KS, USA). La otra muestra se usó para el estudio de hMPV por reacción de polimerasa en cadena previa transcripción reversa (RPC-TR) que fue transportadas a $4{ }^{\circ} \mathrm{C}$ al laboratorio de Infectología y Virología molecular de la Universidad Católica de Chile, donde fueron procesadas. Para el análisis de RPC-TR, la extracción de ARN se realizó con los reactivos High Pure Nucleic Acid (Roche Diagnostic Molecular Biochemicals, Mannheim, Germany) siguiendo las instrucciones de los fabricantes. El procedimiento se realizó según referencia. En el caso de no establecer la etiología se continuó con: estudio de Bordetella pertussis por IFD empleando anticuerpos monoclonales (Accu-MAB TM Plus Canada) y PCR de muestra obtenida por aspirado nasofaríngeo y además serología para Chlamydias (Kit Focus IgG/IgM Asiprod California USA).

Se solicitó a los padres al momento del ingreso del niño firmar un consentimiento informado.

Para el análisis estadístico se utilizó el programa Excell 2003. Se realizó prueba "t" de student para variables cuantitativas y prueba de $\chi^{2}$ para variables cualitativas, se utilizó $\mathrm{p}<0,05$ para considerar la significancia estadística.

\section{Resultados}

Pacientes: Se reclutaron 229 pacientes con una IRAb para el estudio, $124(54,1 \%)$ hombres y $105(45,9 \%)$ mujeres. El promedio de edad fue 30,8 meses (rango: 23 días-166 meses, mediana: 16,6 meses). Menores de 24 meses un $62 \%$ de los pacientes (Tabla 1). Comorbilidad presente en 134 pacientes (58\%); lo más frecuente fue el síndrome bronquial obstructivo recurrente (SBOR) en 69 pacientes $(30 \%)$ (Tabla 2). La duración promedio de la hospitalización fue 7,7 días (rango 1-46, mediana: 6 días). Recibieron tratamiento con antibióticos 141 pacientes $(62 \%)$. Presentaron insuficiencia respi-

Tabla 1. Distribución por edad de 229 niños con infección respiratoria aguda baja

\begin{tabular}{ccc}
\hline Meses & n & \% \\
\hline $0-12$ & 96 & 42 \\
$12-24$ & 45 & 20 \\
$25-60$ & 51 & 22 \\
$>60$ & 37 & 16 \\
Total & 229 & 100 \\
\hline
\end{tabular}

Rev Chil Enf Respir 2008; 24: 107-112 
Tabla 2. Comorbilidad de los pacientes con infección respiratoria aguda baja

\begin{tabular}{lrc}
\hline Comorbilidad & n & \% \\
\hline SBOR & 69 & 30 \\
RNPT & 35 & 15 \\
Síndrome de Down & 10 & 4 \\
Cardiopatías & 7 & 3 \\
DBP & 7 & 3 \\
Neurológico & 5 & 2 \\
DPC & 1 & 0,4 \\
\hline
\end{tabular}

SBOR: Síndrome bronquial obstructivo recurrente; RNPT: Recién nacido de pretérmino; DBP: Displasia broncopulmonar; DPC: Daño pulmonar crónico

Tabla 4. Complicaciones de las infecciones respiratorias bajas graves en niños

\begin{tabular}{lcc}
\hline Complicaciones & n & \% \\
\hline Shock & 25 & 11 \\
Ruptura alveolar & 12 & 5 \\
SIRS & 12 & 5 \\
Fallecido & 1 & 0,4 \\
Total & 50 & 22 \\
\hline
\end{tabular}

SIRS: Síndrome de respuesta inflamatoria sistémica
Tabla 3. Diagnóstico de egreso

\begin{tabular}{lrr}
\hline Diagnóstico & n & \% \\
\hline Laringitis & 21 & 9 \\
Bronquiolitis & 38 & 17 \\
SBO & 48 & 21 \\
Neumonía & 122 & 53 \\
Total & 229 & 100 \\
\hline
\end{tabular}

SBO: Síndrome bronquial obstructivo

Tabla 5. Etiología de las IRAb en pacientes hospitalizados

\begin{tabular}{lrr}
\hline Agente etiológico & $\mathbf{n}$ & $\boldsymbol{\%}$ \\
\hline Virus & 122 & 53 \\
Bacterias & 33 & 15 \\
Desconocidos & 74 & 32 \\
Mixtos & 10 & 4 \\
Total & 229 & 100 \\
\hline
\end{tabular}

Tabla 6. Etiologías de las IRAb estratificadas por edad

\begin{tabular}{cccccc}
\hline Edad (meses) & Total & Virus & Bacterias & Mixtos & Etiología demostrada \\
\hline 0 a 12 & 96 & $66(68)^{*}$ & $10(10)$ & $4(4)$ & $76(79)^{*}$ \\
12 a 24 & 45 & $27(60)^{*}$ & $5(11)$ & $3(6)$ & $32(71)$ \\
25 a 60 & 51 & $24(47)$ & $6(12)$ & $1(2)$ & $30(59)$ \\
$>60$ & 37 & $5(14)$ & $12(32)$ & $1(3)$ & $17(46)^{*}$ \\
Total & 229 & $122(53)$ & $33(14)$ & $9(4)$ & $155(67)$ \\
\hline
\end{tabular}

* Existe asociación estadísticamente significativa entre infección por virus y edad menor de 24 meses. Entre paréntesis los \%.*Diferencia estadísticamente significativa

ratoria grave $84(37 \%)$ pacientes. Se trataron con ventilación mecánica invasiva (VMI) 30 , no invasiva (VMNI) 39 y con ambas modalidades (VMI/VMNI) 15 pacientes. El diagnóstico de egreso más frecuente fue neumonía con o sin derrame pleural asociado en el 53\% (Tabla 3). Se presentaron complicaciones en un $22 \%$ y un paciente falleció (Tabla 4).

Etiología: Uno o más potenciales agentes causales se detectaron en 155 de los 229 pacientes (68\%) (Tabla 5). Evidencia de infección viral fue demostrada en un 53\% (122 pacientes), bacteriana en un 15\% (33 pacientes, asociada a un virus en 9 casos). No se logró demostrar la causa de la IRAb en un $32 \%$ (74 pacientes). La infección por virus fue significativamente más frecuente en los pacientes menores de 24 meses de edad (66\%) que en los pacientes mayores de 24 meses $(33 \%)$ y la proporción de infecciones por bacterias aumenta en los mayores (Tabla 6). Se aisló un virus respiratorio en 137 pacientes (Tabla 7). De los 
Tabla 7. Etiología IRAb en niños hospitalizados

\begin{tabular}{lcc}
\hline Virus & $\mathbf{n}$ & $\mathbf{\%}$ \\
\hline Virus respiratorio sincicial & 88 & 63,8 \\
Metapneumovirus & $24 *$ & 17,4 \\
Parainfluenza & 11 & 10,9 \\
Tipo 1 & 2 & \\
Tipo 2 & 2 & \\
Tipo 3 & 7 & \\
Influenza tipo A & 5 & 3,6 \\
Adenovirus & 9 & 6,5 \\
Total & 137 & 60 \\
& & \\
Bacterias (\%) & & \\
Streptococcus pneumoniae & 12 & 26,1 \\
Mycoplasma pneumoniae & 14 & 30,4 \\
Bordetella pertussis & 8 & 17,4 \\
Chlamydia pneumoniae & 1 & 2,2 \\
Total & 35 & 15 \\
\hline
\end{tabular}

*50\% aislado asociado a otro virus respiratorio
5 virus estudiados, VRS resultó ser el más frecuentemente aislado ( 88 pacientes). Se presentó en pacientes más pequeños y en relación a los otros virus su evolución fue más grave (Tablas 8 y 9). Se demostró hMPV en 24 pacientes, el $50 \%$ aislado sólo y el otro $50 \%$ aislado junto a otro virus respiratorio. La asociación entre hMPV y otro virus no significó una evolución más grave del paciente en términos de promedio de días de hospitalización, ventilación mecánica, uso de vasoactivos (Tabla 10). Una infección bacteriana fue demostrada en 33 pacientes (15\%). Las más frecuentes fueron Mycoplasma pneumoniae en 14 y Streptococcus pneumoniae en 12 pacientes (aislamiento en hemocultivos y cultivo de líquido pleural). La proporción de niños con un agente identificado disminuye significativamente al aumentar la edad (Figura 1). El diagnóstico etiológico fue documentado en el $79 \%$ de los pacientes menores de 12 meses y sólo en el $46 \%$ de los mayores de 60 meses de edad.

Tabla 8. Evolución de la infección respiratoria aguda según etiología

\begin{tabular}{|c|c|c|c|c|c|c|}
\hline Patógenos & $\begin{array}{l}\text { xEdad } \\
\text { (meses) }\end{array}$ & $\begin{array}{c}\text { x días } \\
\text { hospitalizado }\end{array}$ & $\begin{array}{l}\text { VMINI } \\
\text { (n) }\end{array}$ & $\begin{array}{l}\mathrm{VM} \\
(\mathrm{n})\end{array}$ & $\begin{array}{c}\text { VMNI/VM } \\
\text { (n) }\end{array}$ & $\begin{array}{r}\text { DVA } \\
\text { (n) }\end{array}$ \\
\hline \multicolumn{7}{|l|}{ Virus } \\
\hline VRS & 13,4 & 9,4 & 25 & 19 & 11 & 12 \\
\hline Metapneumovirus & 20,1 & 7,7 & 4 & 3 & 0 & 3 \\
\hline Parainfluenza 1-3 & 17,3 & 5,1 & 0 & 2 & 0 & 0 \\
\hline Influenza A & 73,8 & 4,5 & 0 & 0 & 0 & 0 \\
\hline Adenovirus & 33,5 & 14 & 2 & 2 & 1 & 2 \\
\hline \multicolumn{7}{|l|}{ Bacterias } \\
\hline Streptococcus pneumoniae & 60,3 & 12,3 & 2 & 3 & 2 & 2 \\
\hline Mycoplasma & 96,0 & 6,4 & 3 & 0 & 0 & 0 \\
\hline Bordetella pertussis & 11,4 & 10,2 & 3 & 1 & 1 & 0 \\
\hline
\end{tabular}

n: $n^{\circ}$ de pacientes; DVA: Drogas vasoactivas; x: Promedio; VM: ventilación mecánica; VMNI: ventilación mecánica no invasiva

Tabla 9. Evolución IRAb por VRS versus otros virus

\begin{tabular}{lrcl}
\hline Variables & VRS & $\begin{array}{c}\text { Otros } \\
\text { virus }\end{array}$ & p \\
\hline Edad (meses) & 13,4 & 30,4 & $0,01^{*}$ \\
Días de hospitalización & 9,3 & 7,3 & 0,06 \\
Días en VMI & 2,1 & 1,3 & 0,13 \\
Días en VMNI & 1,2 & 0,6 & $0,04^{*}$ \\
Días con DVA & 0,9 & 0,8 & 0,40 \\
\hline
\end{tabular}

DVA: drogas vasoactivas; *Diferencia estadísticamente significativa; VM: ventilación mecánica; VMNI: ventilación mecánica no invasiva
Tabla 10. Evolución IRAb por hMPV

\begin{tabular}{lrcc}
\hline Variables & hMPV & $\begin{array}{c}\text { hMPV+ } \\
\text { otro virus }\end{array}$ & $\mathbf{p}$ \\
\hline Edad (meses) & 20,2 & 18,0 & 0,38 \\
Días de hospitalización & 7,8 & 7,7 & 0,26 \\
Días en VMI & 1,8 & 1,2 & 0,31 \\
Días en VMNI & 1,3 & 0,8 & 0,21 \\
Días con DVA & 1,0 & 0,1 & 0,07 \\
\hline
\end{tabular}

VM: ventilación mecánica; VMNI: ventilación mecánica no invasiva; DVA: drogas vasoactivas 


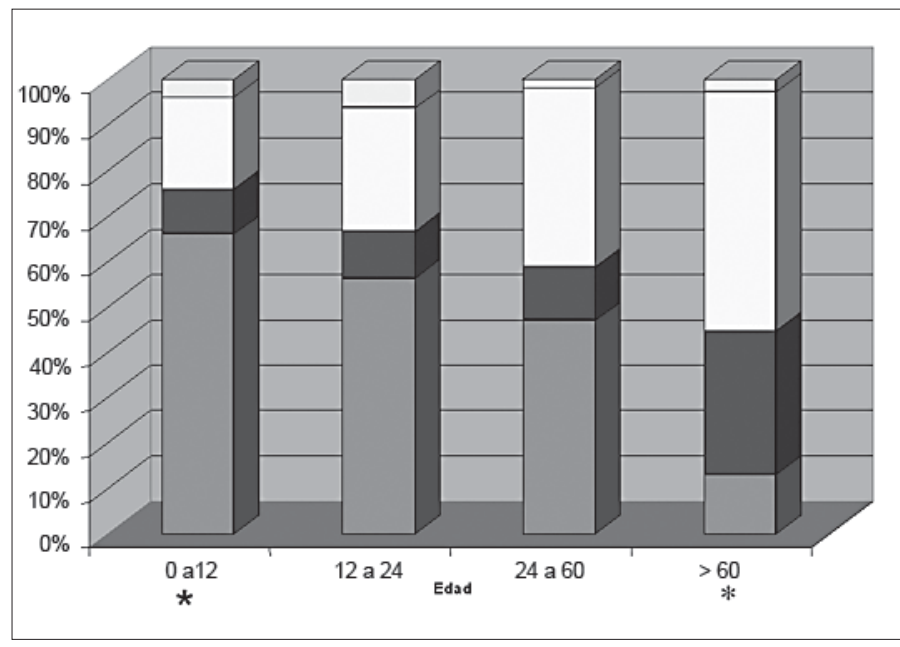

Figura 1. Etiología de las infecciones respiratorias bajas de acuerdo a la edad del paciente expresada en meses. En cada columna se representa el \% de causa viral (en gris), bacteriana (en negro), desconocida (en blanco) y mixta (superior en blanco). $*=p<0,05$ en ambos grupos etarios con etiología demostrada.

\section{Discusión}

La aplicación en forma sistemática de este protocolo de estudio etiológico que combinó test microbiológicos, serológicos, y bioquímicos a 229 niños que fueron hospitalizados por una IRAb en la UPCPCSM permitió establecer un posible agente causal en un $68 \%$ de los casos. El estudio fue realizado en un período de dos años consecutivos para evitar modificaciones en los resultados debido a la posible ocurrencia de un brote epidémico de algún agente en particular.

La infección por un virus fue demostrada en el $60 \%$. Concordante con otras publicaciones ${ }^{1,5,14}$, nuestro trabajo confirma, la importancia de los virus en la etiología de la IRAb grave en niños, principalmente en los menores de 24 meses de vida, y el impacto que la infección por VRS tiene en la frecuencia y gravedad de las IRAb. El 63\% de los pacientes con una infección por VRS presentó insuficiencia respiratoria grave que requirió ventilación mecánica invasiva o no invasiva. El aislamiento de hMPV en 24 pacientes, no sólo certifica la circulación de este virus en nuestro medio si no que lo ubica en el segundo lugar en importancia. Se reconoce de hMPV ${ }^{6-}$ 9,17,18 lo siguiente: distribución mundial, estacionalidad y síndromes clínicos semejantes a los producidos por el VRS. Su rol como copatógeno no está completamente definido; si la infección mixta por VSR y hMPV se asocia o no a una evolución más grave que la observada sólo por VRS no se conoce con certeza. Algunas publicaciones $^{6}$ demuestran para esta asociación un riesgo significativamente mayor de ingreso de niños a UTI para ventilación mecánica. En nuestro trabajo en el $50 \%$ de los casos el hMPV fue aislado junto a otro virus principalmente VRS. La coinfección no significó una evolución más grave.

La infección bacteriana más frecuente fue por Mycoplasma pneumoniae en 14 pacientes. El diagnóstico se estableció con la determinación de anticuerpos de tipo $\operatorname{IgM}$. En niños y adolescentes una muestra simple de IgM puede ser considerada diagnóstica en la mayoría de los $\operatorname{casos}^{10}$. Típicamente aumenta dentro de los primeros 7 días de la infección y aparece casi dos semanas antes que la IgG. En adultos por posibles infecciones previas es necesario el aumento de 4 veces en el título de anticuerpos en suero recolectado en fase aguda y convaleciente con intervalo de 2 a 4 semanas para confirmar una infección aguda. La infección por S. pneumoniae fue demostrada mediante el cultivo de este agente en sangre y líquido pleural. La bacteremia en neumonía por neumococo es de baja frecuencia y el uso de antibióticos antes de la hospitalización disminuye considerablemente el rendimiento del cultivo de líquido pleural ${ }^{1,5}$. Esto explica el bajo porcentaje de infección por $S$. pneumoniae encontrada en nuestro trabajo. No disponemos de exámenes serológicos y la determinación de antígeno en orina en los niños es considerada poco específica. El $62 \%$ de los pacientes fue tratado con antibióticos durante la hospitalización, a pesar, que se demostró infección bacteriana sólo en el 11\%. Esta decisión fue tomada considerando elementos clínicos y de laboratorio con diferente valor predictivo para etiología 
bacteriana: shock, sepsis y derrame pleural son elementos más consistentes que el aumento de la PCR, consolidación en la Rx o progresión de los infiltrados radiológicos, látex en orina positivo para neumococo y cultivo de secreción tomado a través del tubo endotraqueal en los pacientes sometidos a ventilación mecánica. La estandarización de esta última técnica con cultivos cuantitativos podría guiar en forma más racional el uso de los antibióticos. Esto debería ser evaluado y validado en futuros trabajos.

Existe evidencia creciente que muchas de las IRAb de los niños pueden ser causadas por más de un agente patógeno. Se han comunicado infecciones mixtas hasta en un $35 \%$ de los casos. En nuestro trabajo, la infección mixta virus/bacteria pudo ser demostrada sólo en 9 pacientes (4\%) cifra notoriamente inferior a la reportada en la literatura debido probablemente a las limitaciones en los métodos de identificación de bacterias de nuestro estudio ya descritas y también por el diseño del estudio que establecía investigar Bordetella pertussis y Chlamydias en forma secuencial, es decir, sólo si la primera parte del estudio resultaba negativa.

En conclusión, el protocolo de estudio etiológico propuesto permitió determinar el posible agente causal en el $68 \%$ de los casos de esta cohorte. Se confirma el rol de los virus en la IRAb grave de los niño. Sin embargo, la identificación de un virus no descarta la coexistencia de una bacteria por lo que es necesario disponer de mejores métodos de diagnóstico bacteriológico validados que determinen el uso oportuno y racional de antibióticos en estos niños.

\section{Bibliografía}

1.- MCINTOSH K. Current Concepts: CommunityAcquired Pneumonia in Children. N Engl J Med 2002; 346: 429-37.

2.- MICHELOW I, OLSEN K, LOZANO J, ROLLINS N, DUFFY L, ZIEGLER T, et al. Epidemiology and Characteristics of Community-Adquired Pneumonia in Hospitalized Children. Pediatrics 2004; 113: 701-7.

3.- JUVÉN T, MERTSOLA J, WARIS M, LEINONEN M, MEURMAN O, ROIVAINEN M, et al. Etiology of community-adquired pneumonia in 254 hospitalized children. Pediatr Infect Dis J 2000; 19: 293-8.

4.- TSOLIA M, PSARRAS S, BOSSIOS A, AUDI H,
PALDANIUS M, GOURGIOTIS K, et al. Etiology of Community-Adquired Pneumonia in Hospitalizad School-Age Children:Evidence for High Prevalence of Viral Infections. CID 2004; 39: 681-6.

5.- BRITISH THORACIC SOCIETY. Guidelines for the management of community-adquired pneumonia in childhood. Thorax 2002; 57 (Suppl 1).

6.- PRINCIPI N, BOSIS S, ESPOSITO S. Human metapneumovirus in paediatric patient. Clin Microbiol Infect 2006; 12: 301-8.

7.- ESPER F, BOUCHER D, et al. Human Metapneumovirus in the United States: Clinical Manifestations Associated with a Newly Emerging Respiratory Infection in Children. Pediatrics 2003; 111: 1407-10.

8.- VAN BURIK J. Human Metapneumovirus: Important but Not Currently Diagnosable. Ann Intern Med. 2006; 144: 374-5.

9.- MCINTOSH K, MCADAM A. Human Metapneumovirus-An Important New Respiratory Virus. N Engl J Med 2004; 350: 431-3.

10.- WALTES K. New Concepts of Micoplasma pneumoniae Infections in Children. Pediatr Pulmonol 2003; 36: 267-78.

11.- BLACK S, SHINEFIELD H, et al. Efficacy, safety and immunogenicity of heptavalent pneumococcal conjugate vaccine in children. Pediatr Infect Dis J 2000; 19: 187-95.

12.- WHITNEY C, FARLEY M, HADLER J, HARRISON L, BENNET N, LYNFIELD R, et al. Decline in Invasive Pneumococcal Disease alter the introduction of Protein-Polysaccharide Conjugate Vaccine. N Engl J Med 2003; 348: 1737-46.

13.- WILLSON D, LANDRIGAN C, HORN S, SMOUUT R. Complications in Infants hospitalized for bronchiolitis or respiratory syncytial virus pneumonia. J Pediatr 2003; 143 (5): S142-9.

14.- AVENDAÑO L F, PALOMINO M A, LARRAÑAGA C. Surveillance for respiratory syncytial virus in infants hospitalized for acute lower respiratory infection in Chile (1989 to 2000). J Clin Microbiol 2003; 41: 4879-82.

15.- PALOMINO M A, LARRAÑAGA C, AVENDAÑO L F. Hospital-acquired adenovirus $7 \mathrm{~h}$ infantile respiratory infection in Chile. Pediatr Infect Dis J 2000; 19: 527 31.

16.- PALOMINO M A, LARRAÑAGA C, VILLAGRA E, CAMACHO J, AVENDAÑO L F. Adenovirus and respiratory syncytial virus-adenovirus mixed acute lower respiratory infections in Chilean infants. Pediatr Infect Dis J 2004; 23: 337-41.

17.- LUCHSINGER V, ESCOBAR C, AVENDAÑO L F. Detección de Metapneumovirus humano en niños hospitalizados por infección respiratoria aguda baja en Santiago, Chile. Rev Méd Chile 2005; 133: 1059-64.

18.- PRADO A, PERRET C, MONTECINOS L, VELOZ L, ABARCA K, FERRÉS M, et al. Metapneumovirus humano como causa de hospitalización en niños bajo 3 años de edad, con infección respiratoria aguda, durante el año 2004. Rev Chil Infect 2007; 24: 19-26.
Correspondencia a:

Dr. Jaime Lozano Comparini

Servicio Broncopulmonar

Clínica Santa María

Dirección: Av. Santa María 0410, Providencia, Santiago

E-mail: jlozano@csm.cl 